\title{
Frequency behavior of conformal wire structures
}

\author{
Giovanni Leone ${ }^{1}$, Francesco Mattiello ${ }^{1}$, Giuseppe Ruvio ${ }^{1,2}$, \\ Rocco Pierri ${ }^{1}$ \\ ${ }^{1}$ Dipartimento di Ingegneria Industriale e dell'Informazione, Seconda Università di Napoli, Italy \\ ${ }^{2}$ Antenna \& High Frequency Research Centre, Dublin Institute of Technology, Dublin, Ireland \\ *corresponding author, E-mail: francesco.mattiello@unina2.it
}

\begin{abstract}
Three-dimensional models are built to examine the frequency behavior of wire structures conforming onto nonplanar surfaces. The effects of altered periodicity on frequency selectiveness of the structures are considered. An analogy is drawn with the antenna impedance behavior of a single active wire surrounded by a number of passive similar ones. Numerical simulations are performed for different wire geometries over a conical surface.
\end{abstract}

\section{Introduction}

FREQUENCY Selective Surfaces (FSS) have been studied for the last five decades $[1,2,3]$. Today there are many forms of FSS, due to increasingly stringent performance requirements of recent applications and to the significant improvements in analysis [4]. Two types of FSS behavior are observed, either stop band for structures made with metal strips, or pass band for structures made with slots cut into a metallic screen. The simpler types of FSS are made by a planar two-dimensional array of simple scattering objects. Their transmission and reflection coefficients are depending by the operating frequency, the angle of incidence and the polarization of the incident wave.

In the literature the planar case is well documented and treated. However, although attempts have been made, less attention has been dedicated to the finite size conformal FSS. Some approximate analyses start from the unit cell approach, which is the exact or numerical solution of the scattering of a single element in the planar case, and next move to a numerical analysis either in cylindrical [5] or in conical geometry [6]. In [7] it is investigated the curved strip grating of one-dimensional truncation and numerical solutions are proposed as well as local planar approximations.

More rigorous approaches accounting for mutual coupling between curved and/or conformal array elements are also available. In [8] two methods based on Pocklington's equation for wire objects have been put forward. The method has been applied to finite arrays of dipoles arranged on a cylindrical surface with different radii of curvature and illuminated by an arbitrary incident plane wave field to extract frequency selective characteristics. The scattering integral equations are solved for the induced currents over the objects by an appropriate choice of the basis functions in the spatial [9] or in the spectral [10] domains.

For a finite size and conformal FSS it is not possible to perform a simple analysis, because periodicity holds only partially or does not at all, and the scattering objects are arranged as in a conformal array, so that the entire structure must be analyzed. This entails to perform different time consuming scattering analysis for different plane wave incidence angles for a thorough prediction of its behavior for different observation angles. However in many circumstances it is required only to appreciate the general frequency behavior of the surface and faster approaches can be examined.

In this paper a way to assess the frequency behavior of the entire structure is discussed. The proposed technique is effective since it only requires to analyze a part of the entire FSS as an active transmitting subarray. In [11] some numerical results of this approach about the scattering by conical surfaces have been shown. Hereafter we first introduce a simple electromagnetic model to understand this feature. The approach is tested by numerical simulations of the scattering by planar surfaces composed of metallic wires. Next we are interested not only to straight and curved wires conformal to a conical surface, where periodicity conditions are inapplicable, but also to circular loop wires.

Next Section introduces the approach by referring to a surface made of wires made of Perfect Electrical Conducting (PEC) material. Section 3 shows the numerical evaluation of the antenna impedance of some planar and conformal arrays of wires. In Section 4 the scattering behavior of FSS made of the same wire arrays of the previous Section is computed.

\section{The proposed approach}

Let us consider first a single, not necessarily straight, wire and discuss its interaction with an impinging plane wave $\underline{E}_{i}$ (see Fig. 1). We suppose that a reference section is defined along the wire, so that it can be decomposed into two close wires connected by a short circuit at this reference section. The scattered field is related to the induced current along the wire $I(s)$ along the wire ( $s$ is the abscissa along the wire). Apart from the shape and length of the wire, its behavior depends also on the direction of the impinging field. 
Anyway the induced current can be considered as weighted by the short circuit current $\mathrm{I}_{\mathrm{SC}}$ at the reference section $s=0$, so that $I_{S C}=I(0)$.

By considering the wire as a receiving antenna closed on a short circuit and invoking the Norton equivalent circuit of a receiving antenna, $I_{S C}=V_{O C} / Z_{A}$ where $V_{O C}$ is the induced voltage on the receiving wire antenna at the chosen reference section under open circuit conditions and $Z_{A}$ is the impedance at the reference section of the same wire when acting as an antenna fed at the chosen reference section. In turn, $V_{O C}=h_{e} \cdot E_{i}[11]$ that is the dot product of the impinging electric field, evaluated at the reference section $s=0$, with the vector antenna effective height $\underline{h}_{e}$, which is proportional to the far field radiated by the wire when acting as an antenna.

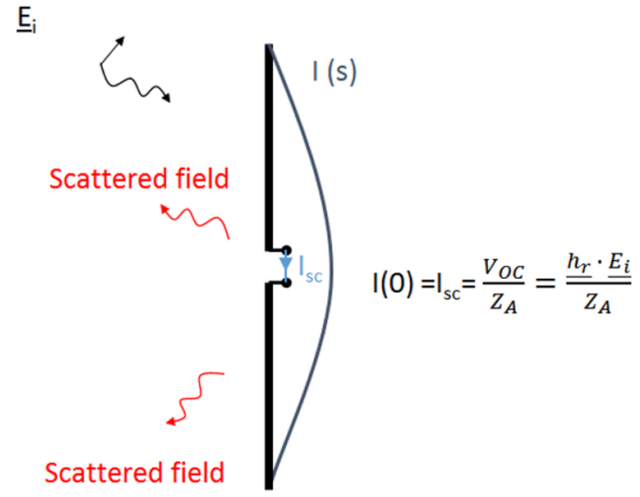

Fig. 1. The receiving antenna scheme

Let us now examine the frequency behavior of the each of the above contributions to the scattered field. When changing the frequency of the impinging field also the induced current on the wire under short circuit condition $I(s)$ varies. However, a mild dependence with frequency can be expected if we do not consider a wide band excitation. The same mild dependence can be expected also for the field radiated by the wire when active, that is for $\underline{h}_{e}$ (and accordingly also for $V_{O C}$ ), since the current excited on the wire antenna by a localized source at the reference section do not vary too much with the excitation frequency within a narrow band. But the situation is remarkably different for $\mathrm{I}_{\mathrm{SC}}$. In fact, the antenna impedance $Z_{A}$ of the wire depends strongly on the frequency also under narrow band excitation. This is especially true for the reactance due to the near fields variations. Therefore, it appears that the frequency behavior of the field scattered by the entire (passive) wire is dominated by the frequency behavior antenna impedance of the wire when excited as an antenna at the chosen reference section.

The same behavior is expected when a wire is surrounded by other similar elements which are not too close each other (about more than half a wavelength apart) and a narrow bandwidth is considered. The critical factor affecting mainly the general frequency behavior of the scattered field is again the antenna impedance of a single active wire (in presence of the other passive ones).

In the following, in order to appreciate this point, we numerically evaluate the antenna impedance of a single wire (surrounded by a number of identical passive wires) for different both straight and curved wire geometries over planar and conical surfaces. Next we compare this frequency behavior of $Z_{A}$ with the backscattered field by the whole surface composed of the same wires.

While the evaluation of the backscattered field requires to consider the entire array structure since the impinging wave illuminates all the wires, the computation of the antenna impedance of a single wire (in presence of the others) may require to consider only a limited part of the structure. In fact, when the wire is excited at its reference section, a current is induced only on a very limited number of the surrounding passive wires due to the mutual coupling between close elements. Accordingly, the antenna impedance evaluation can be performed by considering a subarray of the wires of the entire structure, with obvious advantages with respect to computer time.

\section{Antenna impedance analysis}

Hereafter we consider the following elementary scatterers: a straight wire dipole, a curved wire dipole wire and a circular loop wire, and the following surface geometries: plane and cone. In order to appreciate the proximity effect we compare the antenna impedance behavior (versus frequency) of the active centrally located element when surrounded by other identical passive elements with the case of a single element. The number of close elements has been chosen so that the mutual coupling of further elements does not affect the antenna impedance frequency behavior. The numerical computations concerning the impedance analysis are performed with CST Microwave Studio in the present Section, whereas the scattered power evaluation of radial and curved strips on a cone is performed by the method of moments solution of the relevant integral equations as performed in [11], in order to provide an independent assessment of the approach. It is everywhere supposed that objects are embedded in free space.

\subsection{Straight wires in planar geometry}

For the sake of reference, first we consider a $1.5 \mathrm{~cm}$ long straight wire and a 7x7 array of identical elements, arranged on a plane which are spaced $3 \mathrm{~cm}$ away along the dipole axes and $1.5 \mathrm{~cm}$ along the orthogonal direction (Fig. 2).

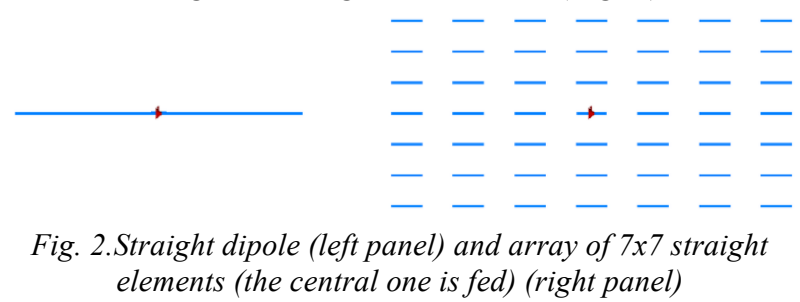

Fig. 3 shows the behavior of the antenna impedance of both the isolated and the wire surrounded by the passive ones in the frequency range 8-16 GHz. 


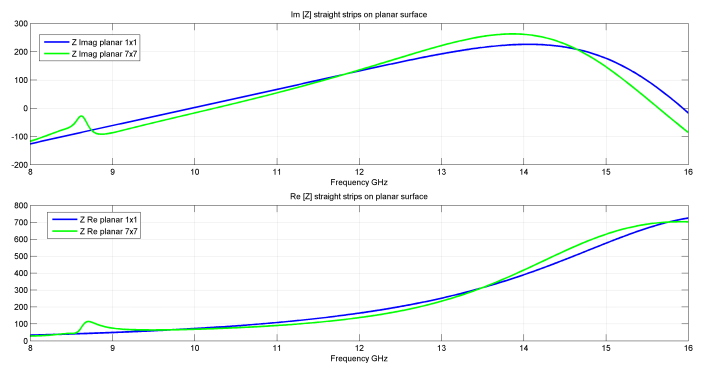

Fig. 3. Imaginary part (top panel) and real part (bottom panel) of $Z_{A}$ of the isolated wire (blue line) and the wire surrounded by identical passive ones (green line)

Since the frequency behavior are very similar a limited effect of the mutual coupling of close elements can be observed. The frequency corresponding to a null in the imaginary part of the antenna impedance does not change appreciably with respect to the case of a single isolated wire since is shifted about $3 \%$ with respect to $9.96 \mathrm{GHz}$.. Accordingly, it can be expected that the frequency of maximum scattering will correspond again to the one where the length of the wire is about $\lambda / 2$.

\subsection{Straight wires in a conical geometry}

Next we consider a $1.5 \mathrm{~cm}$ long straight wire and a $7 \times 7$ array of identical elements, whose centers are spaced $3 \mathrm{~cm}$ away along the dipole axes and $1.5 \mathrm{~cm}$ along the orthogonal direction (Fig. 4). They are arranged on a conformal conical geometry, with apex angle equal to $\pi / 6$.

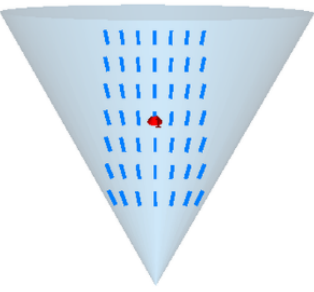

Fig. 4.Straight dipole and array of $7 x 7$ straight elements (the central one is fed)

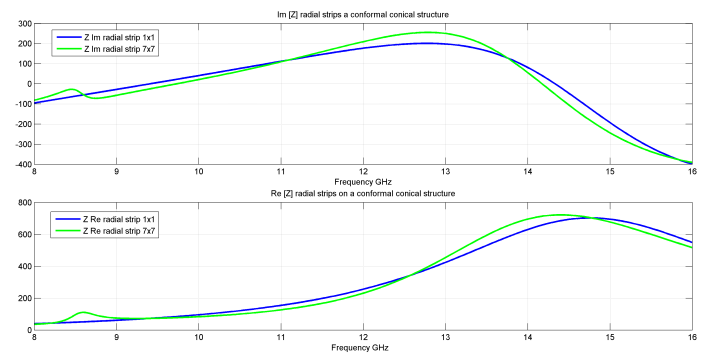

Fig. 5. Imaginary part (top panel), and real part (bottom panel) of $Z_{A}$ of the isolated curved wire (blue line) and the wire surrounded by identical passive ones (green line)

Fig. 5 shows the behavior of the antenna impedance of both the isolated and the wire surrounded by passive ones in the frequency range 8-16 GHz.

It can be appreciated that the arrangement on a conformal surface does not change appreciably the antenna impedance behavior for the considered geometry. In fact the null value of the imaginary part the input impedance in the $7 \times 7$ array, which occurs at $9.7 \mathrm{GHz}$, while for the isolated wire antenna occurs at $9.4 \mathrm{GHz}$. So it is shifted up in frequency about $3 \%$.

\subsection{Curved wires in a conical geometry}

We now move to conformal wires and consider a structure made of circumferential wires, each $1.5 \mathrm{~cm}$ long. They are arranged under an array of $7 \times 7$ identical elements on a conformal conical geometry, with apex angle equal to $\pi / 6$. The wire centers ranges from $4.5 \mathrm{~cm}$ to $13.5 \mathrm{~cm}$ away from the tip of the cone, so that they are $1.5 \mathrm{~cm}$ spaced away along the radial coordinate and are $3 \mathrm{~cm}$ spaced away along each circumference (fig. 6).

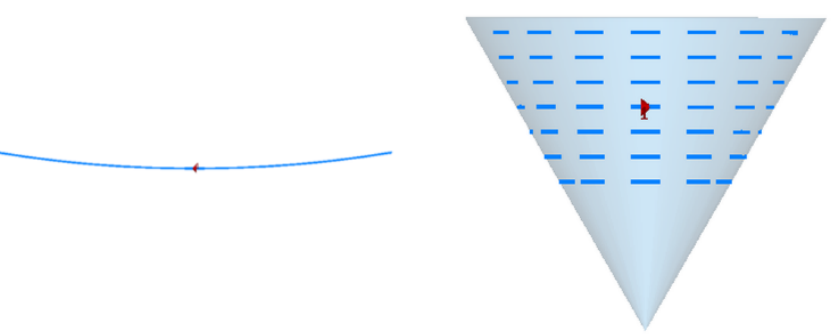

Fig. 6. Curved wire (left panel) and array of $7 x 7$ curved elements (the central one is fed) (right panel)

Fig. 7 shows the behavior of the antenna impedance of both the isolated and the wire surrounded by the passive ones in the frequency range $8-16 \mathrm{GHz}$.

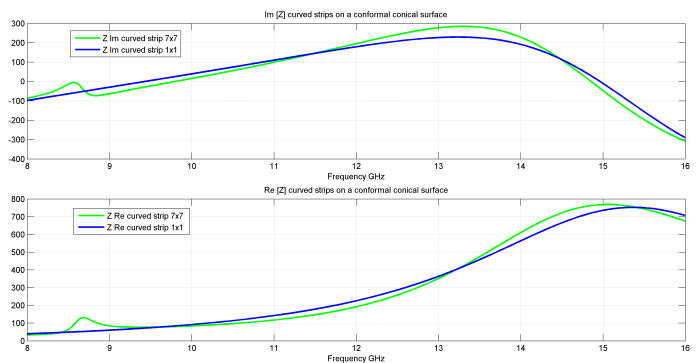

Fig. 7. Imaginary part (top panel), and real part (bottom panel) of $Z_{A}$ of the isolated curved wire (blue line) and the wire surrounded by identical passive ones (green line)

It can be appreciated that in the conformal geometry, for the considered spacing between the elements, the antenna impedance is not affected remarkably by the nonplanar geometrical features. In fact, again, its imaginary part achieves the null value around $9.8 \mathrm{GHz}$, which is shifted up in the $7 \times 7$ array about $4 \%$ with respect to the isolated wire scenario, occurring at $9.43 \mathrm{GHz}$.

\subsection{Circular loop wires in a conical geometry}

In this paragraph we analyze the behavior of a structure made of $3 \mathrm{~cm}$ long circular loops which are made conformal to the same cone as the previous section and compare the behavior of an isolated loop wire with the one of an array of $5 \times 5$ elements. The centers of the loops are $1.5 \mathrm{~cm}$ spaced away in both vertical and horizontal directions (fig. 8). 

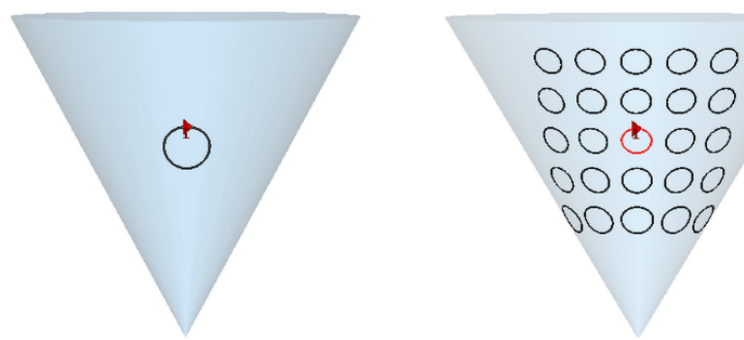

Fig. 8. Curved loop wire (left panel) and array of $5 \times 5$ loop elements (the central one is fed) (right panel)

Fig. 9 shows the frequency behavior of the antenna impedance of both the isolated loop and the loop surrounded by the passive ones in the frequency range $8-16 \mathrm{GHz}$.

Again because of the limited effect of the mutual coupling of close elements, the frequency corresponding to a null into the imaginary part of the antenna impedance, which occurs around $11.27 \mathrm{GHz}$, does not change too much with respect to case of an isolated loop. In particular a 2\% downward shift is observed at $11.05 \mathrm{GHz}$. Accordingly, it can be expected that the frequency of maximum scattering will correspond again to the one where the length of the loop is about $\lambda$.

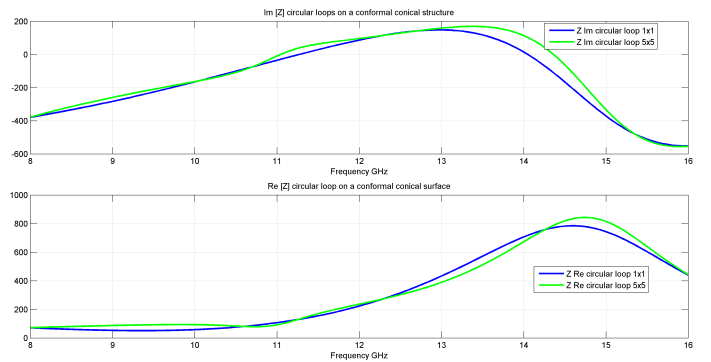

Fig. 9.Imaginary part (top panel), and real part (bottom panel) of $Z_{A}$ of the isolated loop wire (blue line) and the wire surrounded by identical passive ones (green line)

\section{Behavior of scattered field}

This section is devoted to the frequency behavior of the field backscattered by different conformal wire structures, which are built starting from the examples considered in the previous section. In order to appreciate their overall scattering properties, the total scattered power in correspondence of a single plane wave incidence has been numerically evaluated, as in [11] for the radial and curved strips on a conformal conical surface, and using CST Studio for the strip loops on a cone. In all subsequent cases, the plane wave with linear polarization parallel to the orientation of the wire impinges form the $\theta_{i}=\pi / 3, \varphi_{i}=\pi$ direction (that is from a direction normal with respect to the surface of the cone).

\subsection{Straight wires in a conical geometry}

The first geometry stems from the one of Section $\mathbf{3 . 2}$ and is composed of 5 full rows of radial wires, spaced away vertically by $3 \mathrm{~cm}$ and horizontally by $1.5 \mathrm{~cm}$. The total number of wires is 181. (fig. 10).
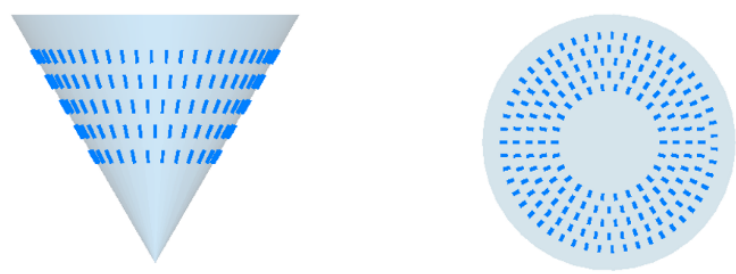

Fig. 10. Structure made of radial strips conformed on a cone: side (left panel) and top (right panel) views

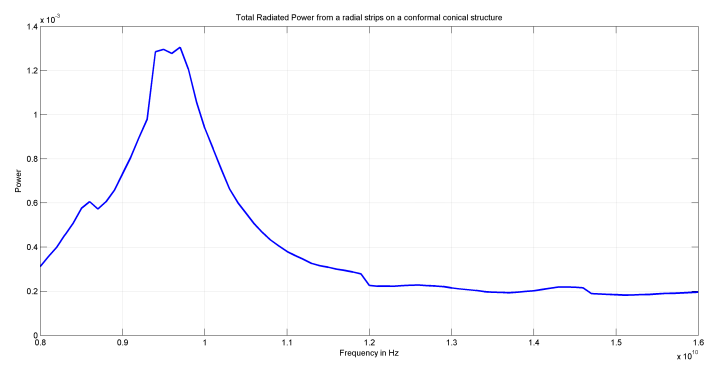

Fig. 11. Total scattered power for plane wave incidence

The numerical results of Fig. 11 shows that the maximum of the scattered power is attained at around $9.6 \mathrm{GHz}$, which well corresponds to the null of the imaginary part of the antenna impedance of structure of Fig. 5.

\subsection{Curved wires in a conical geometry}

This geometry stems from the one of Section $\mathbf{3 . 3}$ and is composed of 5 full rows of circumferential wires, spaced away vertically by $3 \mathrm{~cm}$ and horizontally by $1.5 \mathrm{~cm}$. The total number of wires is 60 (fig. 12)
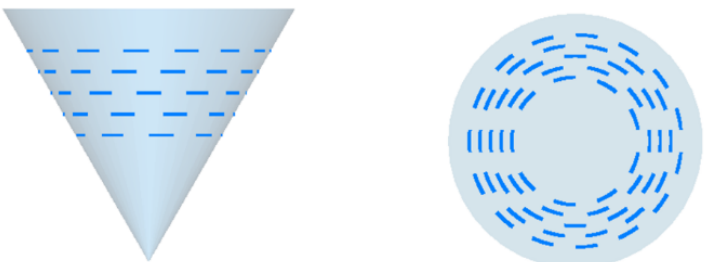

Fig. 12. Structure made of circumferential strips conformed on a cone: side (left panel) and top (right panel) views

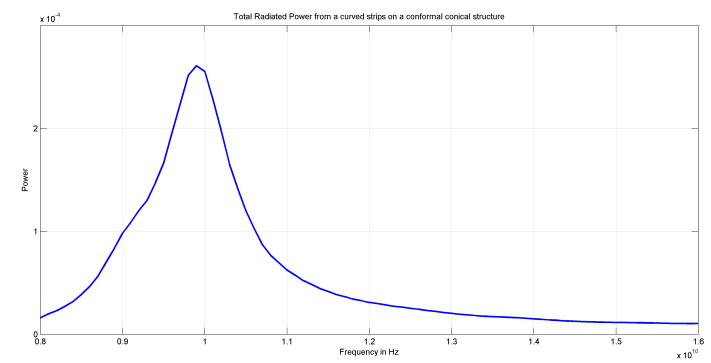

Fig. 13. Total scattered power for plane wave incidence

Again the maximum of the scattered power (Fig. 13) is attained in correspondence of a null of the imaginary part of the antenna impedance of structure of Fig. 7, at around 9.9 $\mathrm{GHz}$. 


\subsection{Circular loop wires in a conical geometry}

In this example it is considered a structure composed of 5 full rows of circular loops, with a diameter of $3 \mathrm{~cm}$ and spaced apart by $1.5 \mathrm{~cm}$ both vertically and horizontally. The total number of wire loops is 85 (fig. 14)

In this case the maximum of the scattered power (fig. 15) is achieved at $11.2 \mathrm{GHz}$. This result is consistent with the accuracy of the numerical computation of the imaginary part of the antenna impedance of Section 3.4, where a low frequency shift has been observed between the isolated and the $5 \times 5$ structures.
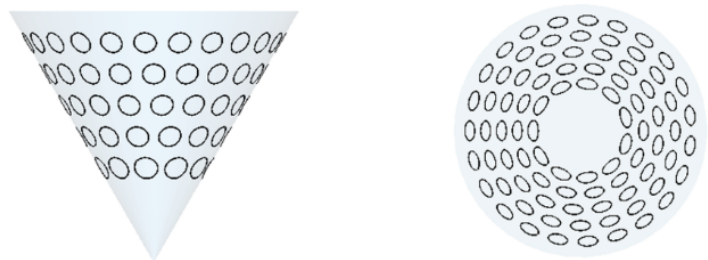

Fig. 14. Structure made of circular loop wires conformed on a cone: side (left panel) and top (right panel) views

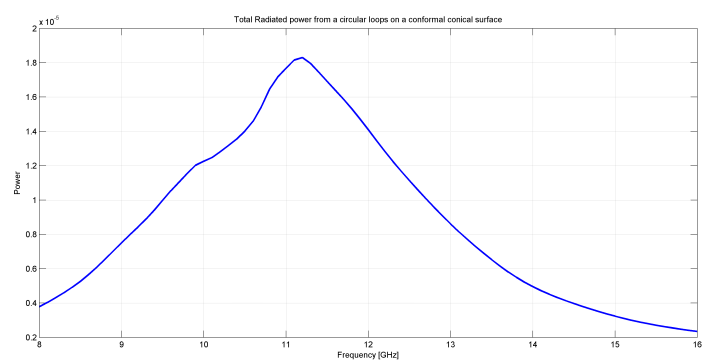

Fig. 15. Total scattered power for plane wave incidence

\section{Spacing influence}

A closer spacing between the wires will imply the insurgence of stronger mutual coupling, which it can be expected to affect the frequency behavior of the power scattered by the strip geometry under different points of views. First of all it makes the antenna impedance a faster function of the frequency. For example, this can be appreciated by the computation of the impedance function for closer strip elements on the conformal conical surface of Section 3.2.

In addition it makes also the currents induced on the passive strips of Section III a faster function of the frequency so affecting the vector antenna effective height $\underline{h}_{e}$, and consequently the induced voltage on the receiving wire antenna at the chosen reference section under open circuit conditions.
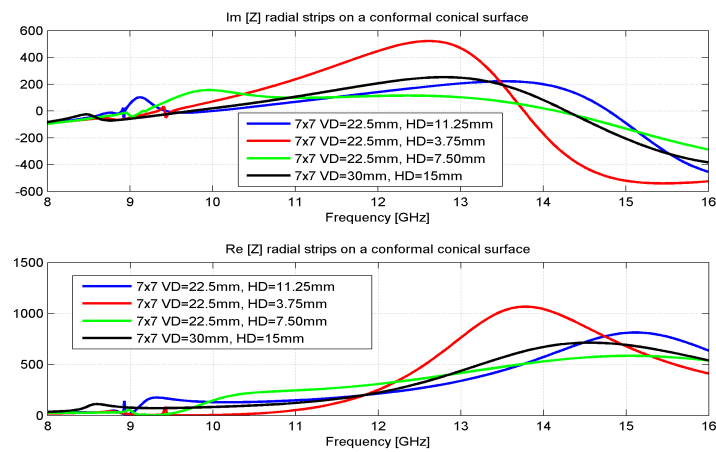

Fig. 16. Imaginary part (top panel), and real part (bottom panel) of $Z_{A}$ of a wire surrounded by identical passive ones for the geometry of Fig. 4 with different spacings. (VD stands for the Vertical Distance and HD is for Horizontal Distance between the wire centers).

Finally it makes also the induced current on the wire under short circuit condition a faster function of the frequency. Unfortunately it is not easy to show in a synthetic way both latter variations. However the overall effect to be expected by the combination of all those different frequency variabilites is that the FSS behavior of the structure is lost. This can be readily appreciated by plotting the total scattered power by the sample wire structure of Fig. 17, composed of 5 full rows of radial wires, spaced away vertically by 2.25 $\mathrm{cm}$ and horizontally by $0.375 \mathrm{~cm}$.

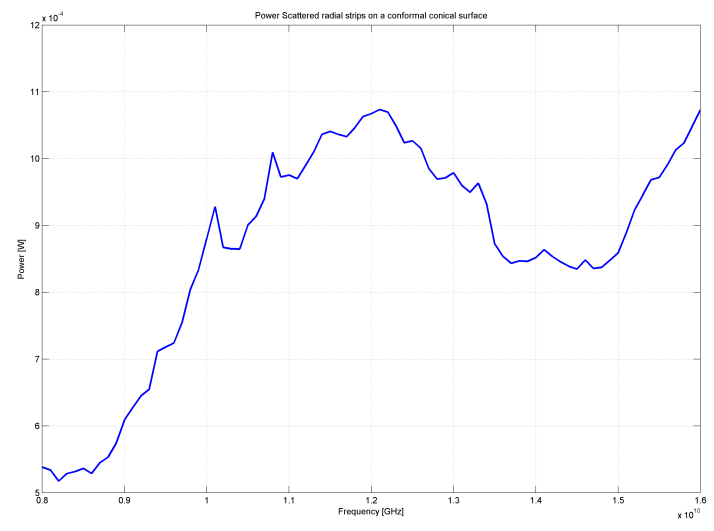

Fig. 17. Total scattered power for a plane wave incidence.

Now, in fact, the overall dynamic of the power scattered in the observation frequency band is 1 to about 0.5 with respect to the maximum, that is a nearly constant frequency behavior. So it can be concluded that at strips spacings closer than the ones considered in Section III and IV, mutual coupling badly affects the FSS behavior of the conformal structure.

\section{Conclusions}

The prediction of the frequency behavior of nonplanar wire FSS structures is difficult because periodicity lacks. By founding on the scattering properties of a single wire element (surrounded by the other similar ones), a simplified model of the frequency behavior is drawn. It is observed that this depends on the antenna impedance of the same 
wire when acting as a source, in a way similar to the active impedance of an array. A further advantage concerns the possibility not to consider the whole structure, but only a limited part of it, with obvious reduction of the time of the numerical simulation. For the considered cases the frequency selectivity of a conformal wire structure is observed to be very similar to the corresponding straight wire one.

\section{Acknowledgements}

This work was supported by POR Campania FSE 2007 2013 "Materiali e Strutture Intelligenti" MASTRI Excellence Network"

\section{References}

[1] B. A. Munk, Frequency Selective Surfaces: Theory and Design, John Wiley \& Sons Inc, New York, 2000.

[2] T. K. Wu, Frequency Selective Surface and Grid Array, John Wiley \& Sons Inc, New York, 1995.

[3] F. Mattiello, G. Leone, G. Ruvio, Analysis and Characterization of Finite-size Curved Frequency Selective Surfaces, Studies in Engineering and Technology, Redfame Publishing, 2015

[4] R. U. Nair, R. M. Jha, "Electromagnetic Design and Performance Analysis of Airborne Radomes: Trends and Perspectives", IEEE Antennas and Propagation Magazine, Vol. 56, No.4, pp. 276- 298, August 2014.

[5] A. Dalkilic, L. Alatan, C.B. Top, "Analysis of conformal frequency selective surface radome", 8th European Conference on Antennas and Propagation (EuCAP), The Hague, The Netherlands, pp. 469-472, April 2014.

[6] H. Zhou, S. Qu, B. Lin, J. Wang, H. Ma, Z. Xu, W. Peng, P. Bai, "Filter-antenna of conical FSS radome and monopole antenna", IEEE Transactions on Antennas and Propagation, vol 60, n. 6, pp 3040-3045, June 2012.

[7] T. Cwik, R.Mittra, "The Effects of the Truncation and Curvature of Periodic Surfaces: A Strip Grating", IEEE Transactions on Antennas and Propagation,vol36, n. 5, pp612-622, May 1988.

[8] E.A. Parker, B. Philips, R.J. Langley, "Analysis of Coupling Between a Curved FSS and an Enclosed Planar Dipole Array", IEEE Microwave and Guided Wave Letters, Vol. 5, N. 10. Oct. 1995.

[9] C. Pelletti, G. Bianconi, R. Mittra, A. Monorchio, "Analysis of Finite Conformal Frequency Selective Surfaces via the Characteristic Basis Function Method and Spectral Rotation Approaches", IEEE Antennas and Wireless Propagation Letters, vol. 12, pp. 1404 - 1407, 2013.

[10] Sipus, Z., Bosiljevac, M., "Efficient analysis of curved frequency selective surfaces", 3rd European Conference on Antennas and Propagation, 2009. EuCAP 2009, Berlin, Germany, pp. 2726 - 2730, March 2009.

[11] G. Leone, F. Mattiello, G. Ruvio, R. Pierri, "Behaviour of conformal conical Frequency Selective Surfaces",
Progress in Electromagnetics Research Symposium (PIERS) 2015, Prague, July 2015. 